Instituto Internacional de Investigación y Desarrollo Tecnológico Educativo INDTEC, C.A.

DOI: https://doi.org/10.29394/Scientific.issn.2542-2987.2021.6.22.5.96-116

OAl-PMH: http://www.indteca.com/ojs/index.php/Revista Scientific/oai

Artículo Original / Original Article

\title{
Principales dificultades para el diagnóstico del TDAH en niños en Ecuador
}

Autoras: Estefanía Isabel Lara Orozco Universidad Internacional SEK, UISEK eilara.mpp@uisek.edu.ec

Quito, Ecuador https://orcid.org/0000-0002-9852-9644

Gabriela Alexandra Pazmiño Márquez Universidad Internacional SEK, UISEK gpazminom@gmail.com Quito, Ecuador https://orcid.org/0000-0001-7352-7952

Janethcia del Rocío Játiva Morillo Universidad Internacional SEK, UISEK rocio.jativa@uisek.edu.ec Quito, Ecuador https://orcid.org/0000-0001-7235-1924

\section{Resumen}

Se verifican numerosos casos en que los padres de familia, cuidadores o docentes consideran que el diagnóstico, y por ende la propuesta de tratamiento terapéutico, del Trastorno por Déficit de Atención con Hiperactividad (TDAH) en niños ha sido erróneo. La importancia de esta investigación radica entonces en el análisis de los factores que intervienen en el pronunciamiento de este diagnóstico. Se entrevistó a 11 psicólogos clínicos, cinco psicólogos infantiles y psicorehabilitadores, tres psicólogos generales y dos psicólogos educativos, para conocer las aproximaciones clínicas y las herramientas de valoración que emplean los profesionales en psicología para emitir el diagnóstico de TDAH en niños de hasta 12 años. Esta investigación mantiene un enfoque metodológico cualitativo, descriptivo. Se encontró que en nuestro país los profesionales no cuentan con herramientas adecuadas o suficientes para realizar un diagnóstico de TDAH. El escaso apoyo de las instituciones públicas y la falta de directrices a nivel del sistema de educación nacional es parte del problema. Privilegiar el trabajo en equipo, la comunicación con docentes y padres de familia y la discusión de resultados a nivel de una red de intervención coordinada, podría representar una solución viable y de largo alcance para favorecer un adecuado abordaje del TDAH.

Palabras clave: TDAH; evaluación; diagnóstico; tratamiento.

Código de clasificación internacional: 6104.02 - Métodos educativos.

\section{Cómo citar este artículo:}

Lara, E., Pazmiño, G., \& Játiva, J. (2021). Principales dificultades para el diagnóstico del TDAH en niños en Ecuador. Revista Scientific, 6(22), 96-116, e-ISSN: 2542-2987. Recuperado de: https://doi.org/10.29394/Scientific.issn.2542-2987.2021.6.22.5.96-116

Fecha de Recepción: 14-01-2021
Fecha de Aceptación: 05-06-2021
Fecha de Publicación: 05-11-2021 
OAl-PMH: http://www.indteca.com/ojs/index.php/Revista Scientific/oai

Artículo Original / Original Article

\section{Main difficulties for the diagnosis of ADHD in children in Ecuador}

\section{Abstract}

There are numerous cases in which parents, caregivers or teachers consider that the diagnosis, and therefore the proposed therapeutic treatment, of Attention Deficit Hyperactivity Disorder (ADHD) in children has been wrong. The importance of this research then lies in the analysis of the factors that intervene in the pronouncement of this diagnosis. Eleven clinical psychologists, five child psychologists and psychologists, three general psychologists and two educational psychologists were interviewed to learn about the clinical approaches and assessment tools used by psychology professionals to make the diagnosis of ADHD in children up to 12 years of age. This research maintains a qualitative, descriptive methodological approach. It was found that in our country professionals do not have adequate or sufficient tools to make a diagnosis of ADHD. The scant support from public institutions and the lack of guidelines at the level of the national education system is part of the problem. Privileging teamwork, communication with teachers and parents and the discussion of results at the level of a coordinated intervention network could represent a viable and far-reaching solution to favor an adequate approach to ADHD.

Keywords: ADHD; evaluation; diagnosis; treatment. International classification code: 6104.02 - Educational methods.

How to cite this article:

Lara, E., Pazmiño, G., \& Játiva, J. (2021). Main difficulties for the diagnosis of ADHD in children in Ecuador. Revista Scientific, 6(22), 96-116, e-ISSN: 2542-2987. Recovered from: https://doi.org/10.29394/Scientific.issn.2542-2987.2021.6.22.5.96-116

Date Received: 14-01-2021
Date Acceptance: 05-06-2021
Date Publication: 05-11-2021 


\section{Introducción}

Nuestra propia práctica profesional nos indica que existen casos cada vez más numerosos de niños que han sido erróneamente diagnosticados con Trastorno por Déficit de Atención con Hiperactividad (TDAH) en algún momento de su escolaridad. Asimismo, para Ramos, Bolaños y Ramos (2015): la elevada prevalencia de TDAH en estudiantes ecuatorianos nos alerta acerca de una mayor tendencia de los profesionales en psicología a emitir este diagnóstico en los últimos años.

Diremos de entrada que las fronteras entre el trastorno y el comportamiento normal del niño no logran situarse con facilidad en todos los casos. En consecuencia, muchos profesionales encuentran dificultades al momento de emitir un diagnóstico formal de la conducta del niño, puesto que los problemas atencionales o de aprendizaje no siempre aparecen en primer plano. En muchas ocasiones, el cuadro clínico se describe en términos de la imposibilidad del niño de tolerar la frustración, de respetar los límites y las reglas, de reconocer a las figuras de autoridad, e inclusive se consignan cada vez con más frecuencia comportamientos agresivos, violentos o delictivos en los niños con esta presunción diagnóstica.

En el contexto ecuatoriano, la respuesta del sistema escolar y familiar ante esta problemática continúa siendo el ejercicio de la violencia hacia los niños, según un estudio reciente del Observatorio Social del Ecuador (OSE, 2019a): "la violencia en la escuela es una norma social que se presenta en varias formas, desde abuso o maltrato físico, verbal hasta psicológico" (pág. 136).

A pesar de que esta práctica se encuentra tipificada en el Código de la Niñez y Adolescencia (2003): como una forma de maltrato, muchos de los padres de niños concernidos por este diagnóstico afirman que recurren a castigos físicos como una medida de corrección ante las conductas que consideran inapropiadas e inclusive autorizan a priori al maestro para que 
emplee esta misma medida con sus hijos en la escuela, según se afirma en el mencionado estudio.

A pesar de los objetivos de mayor equidad y calidad educativa planteados por el Estado a través del Plan Decenal de Educación 2006-2015 (PDE), según el Observatorio Social del Ecuador (OSE, 2019b): en el Ecuador no se ha desarrollado una política de cuidado de los niños que se cumpla universalmente. Las normativas existentes en las instituciones educativas y los distintos manuales, herramientas y guías emitidas por el Ministerio de Educación (MINEDUC, 2016): exponen que son relativamente inoperantes en el trabajo cotidiano con niños que presentan una sintomatología asociada al TDAH.

En la práctica, los docentes y educadores se encuentran rápidamente excedidos por la alta incidencia de casos de niños con necesidades pedagógicas especiales, y muchos denuncian su imposibilidad de brindar la atención que éstos requieren, lo que de acuerdo con Reza, Solórzano, Erazo, Oñate y Lamingo (2018): ha elevado significativamente la demanda de valoraciones psicológicas especializadas fuera de la institución educativa.

Los profesionales en psicología se encuentran entonces llamados a intervenir en situaciones en las que el fracaso escolar es inminente, ante padres desorientados y, generalmente, sin el acceso a apoyos institucionales públicos ni privados. En este contexto, un diagnóstico emitido sin la justificación necesaria puede conducir a la estigmatización del niño en su medio escolar y social, con consecuencias que trascienden la infancia y la adolescencia. La banalización de esta problemática y, en general, el desconocimiento de los procesos de evaluación y diagnóstico existentes, implican por lo tanto un problema grave en el trabajo clínico con los niños y las familias.

A pesar de que el Trastorno por Déficit de Atención con Hiperactividad (TDAH) cuenta con numerosos estudios en el plano psicoterapéutico y ha 
despertado un alto interés entre los investigadores, para Milla (2018): en el Ecuador la evaluación y diagnóstico del TDAH ha sido insuficientemente explorada. Pocos trabajos de investigación dan cuenta de la problemática a nivel local, aun cuando la demanda de atención psicológica por este motivo no ha dejado de aumentar en los últimos años.

Al mismo tiempo, sabemos que el diagnóstico de TDAH supone una dificultad inherente a su categorización misma, implica la presencia de dos polos: uno deficitario en el ámbito de la atención y de la adquisición de destrezas de aprendizaje, y un exceso en el ámbito de la actividad y el movimiento. La Asociación Americana de Psiquiatría (2014a): advierte que "el TDAH frecuentemente se solapa con trastornos que a menudo se consideran "trastornos exteriorizadores", como el trastorno negativista desafiante y el trastorno de conducta" (pág. 32).

Es así que esta condición se sitúa entre los Trastornos del neurodesarrollo y los Trastornos disruptivos, del control de impulsos y de la conducta, en función de los patrones de síntomas, la comorbilidad y los factores de riesgo en común. Refiriéndose específicamente al Trastorno por Déficit de Atención, la Asociación Americana de Psiquiatría (2014b), admite que:

[...] Descubrimientos futuros podrían cambiar la ubicación y el contexto de determinados trastornos y, más aún, que la sencilla organización lineal que mejor se adapta a la práctica clínica podría no captar completamente la complejidad y heterogeneidad de los trastornos mentales (pág. 12).

Nos situamos de hecho en un terreno clínico complejo y heterogéneo. En este sentido, Hidalgo y Sánchez (2013): Si bien se conocen las manifestaciones más comunes del TDAH, mismas que son observables en un primer abordaje (agitación motriz, imposibilidad de concentrarse en una sola tarea, déficit más o menos importante en la capacidad de atención, dificultades 
escolares y de aprendizaje), no existe ningún consenso acerca de las causas de este trastorno.

En cuanto a las técnicas y herramientas de diagnóstico propiamente dichas, se emplean a gran escala protocolos basados en presupuestos neurocientíficos. Parafraseando a Meneres-Sancho, Delgado-Pardo, AiresGonzález y Moreno-García (2015a): mencionaremos en particular los Test de ejecución continua (Continuous Performance Test) que han sido establecidos como instrumentos complementarios de evaluación y diagnóstico del TDAH y consisten en la administración de pruebas objetivas para evaluar: 1). Atención; 2). Velocidad de respuesta; 3). Resistencia a las distracciones; y 4). Capacidad de inhibición.

Por otra parte, para Benedet (2002): se considera a la capacidad de atención como un factor primordial para el correcto funcionamiento cognitivo del niño, debido a que asegura dos funciones básicas: mantener el estado de alerta del sistema cognitivo y seleccionar la información relevante proporcionada por el ambiente. Por eso el diagnóstico de TDAH se valora a partir de la simple observación de problemas de atención, tales como la distracción fácil, la dificultad para terminar las tareas propuestas o la verificación de una gran cantidad de errores al resolverlas. Interpretando a Manrique (2019): la evitación de las tareas y actividades que demandan esfuerzo es otro de los signos más relevantes en las primeras observaciones que se consignan en el ámbito escolar y familiar.

En general, se considera que la atención en los niños con una presunción de TDAH suele ser dispersa y tiende a alterarse ante casi cualquier estímulo. Señalando a Zuluaga y López (2017): incluso el niño hiperactivo no puede establecer de manera adecuada un orden de prioridades entre los estímulos que se le presentan.

Podemos entonces relacionar la elevada prevalencia de este diagnóstico con la contigüidad que existe entre los síntomas considerados 
como patológicos con la conducta normal de un niño sano en edad escolar. En esa misma línea, Claro (2015): plantea que, para corregir esta problemática, un amplio rango de investigaciones en psicología se ha inclinado al desarrollo de escalas de medición para realizar un diagnóstico de acuerdo con la necesidad del niño y a partir del cual podría establecerse un plan de tratamiento.

A pesar de ello, las dificultades para emitir un diagnóstico acertado prevalecen entre los profesionales ecuatorianos, lo que podría tener relación con el difícil acceso a las escalas de medición existentes, o incluso a las dificultades para adaptarlas al contexto cultural (traducción de los cuestionarios al castellano, condiciones mínimas requeridas para su administración, duración de los procesos de evaluación, entre otros aspectos). Es indudable que a estos factores debe sumarse la necesidad urgente de aportar una mirada más amplia a la psicopatología infantil, y en particular al diagnóstico y tratamiento del TDAH dada su alta incidencia en nuestro país.

El objetivo de esta investigación es entonces determinar si el proceso de evaluación y diagnóstico del TDAH cumple con criterios clínicos que permitan justificar su elevada prevalencia en el Ecuador, a través del análisis de los procedimientos empleados por los profesionales que atienden a niños que presentan una sintomatología relacionada con este trastorno. Se espera conocer la metodología, técnicas, enfoques y procedimientos de diagnóstico empleados en el proceso de tratamiento de problemas atencionales y relacionados a la hiperactividad infantil, de manera que puedan corregirse las tendencias erróneas o ineficaces que se verifican en el proceso diagnóstico.

\section{Metodología}

Participaron en la presente investigación 21 psicólogos ecuatorianos de varias orientaciones teóricas y clínicas que atiendan o han atendido a niños de hasta 12 años y cuyo diagnóstico haya sido Déficit de Atención con 
Hiperactividad (TDAH). Se entrevistaron 11 psicólogos clínicos, cinco psicólogos infantiles y psicorehabilitadores, tres psicólogos generales y dos psicólogos educativos. Los resultados se han analizado entonces a partir de esta población más reducida, pero más específica en cuanto a la problemática que nos ocupa.

Señalaremos que esta investigación mantiene un enfoque metodológico cualitativo, en el que se identifican las diferentes modalidades de diagnóstico que emplean los profesionales especializados en la atención a niños con una presunción diagnóstica de TDAH. Se trata de un estudio descriptivo de las aproximaciones clínicas y de las herramientas de valoración que emplean los profesionales en psicología para emitir este diagnóstico en niños de hasta 12 años.

Se consideran, además del uso de pruebas objetivas, la observación clínica, el lugar que se otorga a la entrevista con padres y docentes, el vínculo que se establece con el paciente, entre otras variables más o menos subjetivas que forman parte de los procedimientos clínicos formales en el trabajo psicoterapéutico con niños. Se emplea una modalidad de estudio de campo, ya que los datos han sido recogidos directamente de los profesionales que participaron en este estudio en función de su enfoque teórico-clínico.

Para el análisis de datos, se emplearon dos variables: 1). la línea de especialización teórico-clínica de los psicólogos participantes; y 2). las herramientas e instrumentos de los que se dispone en función de dicha especialización. Se consideró además que los factores inherentes a la formación del psicólogo y su propia percepción de las conductas "inadecuadas" del niño pueden influir en la forma en que evalúa y diagnostica el TDAH.

A través de este análisis se buscó determinar si el diagnóstico emitido se encuentra suficientemente justificado desde el punto de vista técnico o si responde más bien al prejuicio del terapeuta acerca de la conducta que se 
espera de un niño, a la necesidad de dar respuesta a la demanda de los padres, a su eventual desconocimiento de las herramientas de evaluación existentes o, inclusive, al desconocimiento de las manifestaciones clínicas del TDAH.

La metodología empleada para la recolección de datos consistió en el diseño y aplicación de un cuestionario de 10 preguntas abiertas en el que los participantes describen en detalle el proceso de evaluación y diagnóstico que cada uno de ellos emplea para pronunciarse por el diagnóstico TDAH en niños de hasta 12 años.

Tras la obtención de datos, se procedió a clasificar la modalidad de evaluación y diagnóstico en función del enfoque teórico del profesional, de manera que se recabe información suficiente para determinar la pertinencia de los procesos de evaluación y diagnóstico del TDAH en nuestro país.

\section{Resultados}

Se exponen a continuación los aspectos más relevantes que se obtuvieron a través de la aplicación del cuestionario a cada uno de los participantes en este estudio. Las respuestas se han agrupado posteriormente en el gráfico 1 , gráfico 2 y gráfico 3 , que presentan los principales resultados.

Gráfico 1. Orientación teórico-clínica de los psicólogos que trabajan con niños con TDAH en el Ecuador.

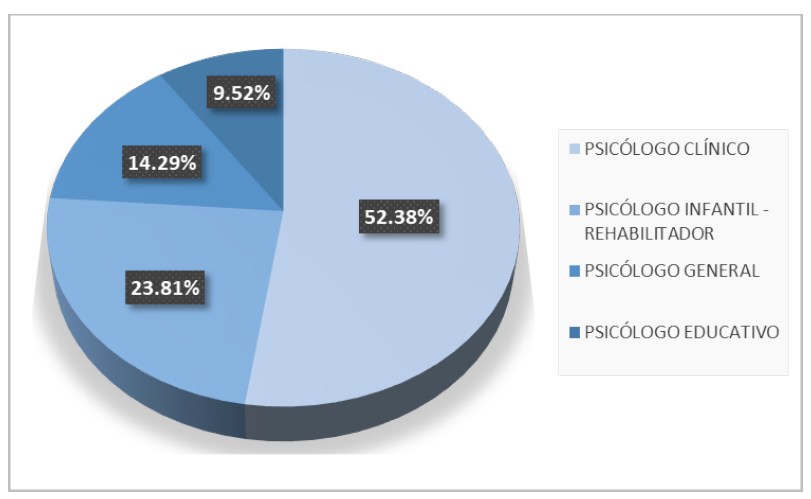

Fuente: Las Autoras (2020). 
El 52\% de la mayoría de los profesionales son psicólogos clínicos, seguidos del 23,81\% psicólogos infantiles - rehabilitadores, 14,29\% psicólogos generales y $9,52 \%$ psicólogos educativos y escolares.

Gráfico 2. Enfoque teórico-clínico de los profesionales que trabajan con niños con TDAH en el Ecuador.

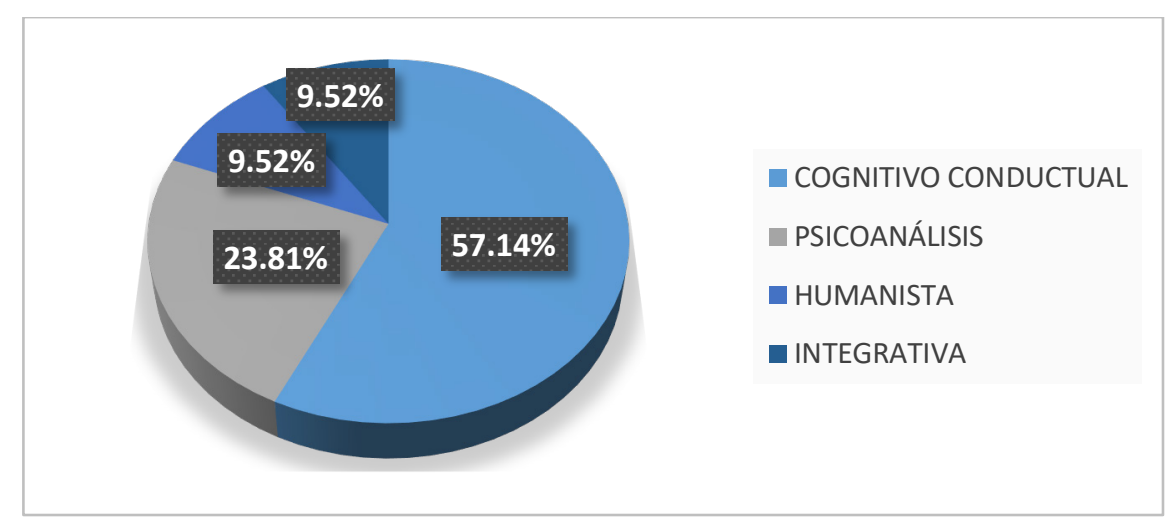

Fuente: Las Autoras (2020).

Los resultados han reflejado que dentro del grupo de psicólogos que trabaja con niños con TDAH, la mayoría del 57,14 emplea un enfoque cognitivo-conductual, seguido por $23,81 \%$ en el enfoque psicoanalítico. Los enfoques humanista e integrativo con $9,52 \%$ aparecen en tercer lugar con un idéntico porcentaje.

Gráfico 3. Proceso de evaluación y diagnóstico del TDAH en niños en el Ecuador.

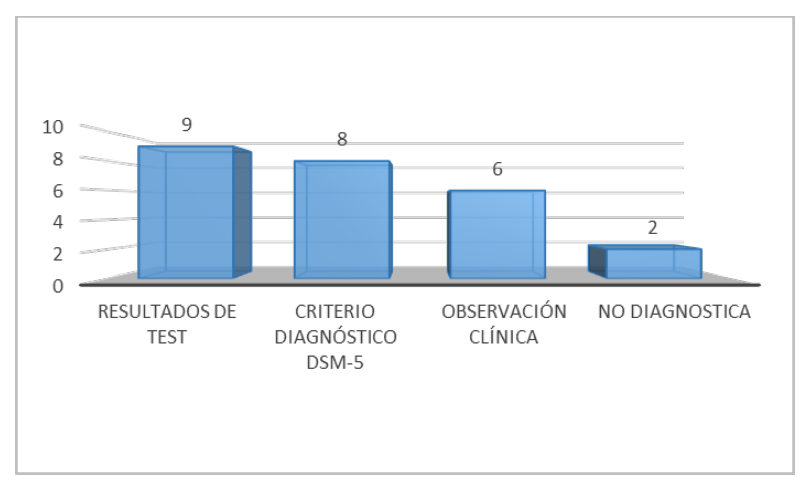

Fuente: Las Autoras (2020). 
Los procesos que los profesionales realizan para emitir el diagnóstico de TDAH, se basan fundamentalmente en los resultados de las baterías de test aplicadas. Se encuentran después los criterios diagnósticos del DSM-5, seguidos de la entrevista psicológica y la observación clínica. Existe un bajo porcentaje de profesionales que no realiza ningún proceso diagnóstico.

En función de los datos obtenidos observamos que no existen consensos entre los profesionales a propósito del diagnóstico del TDAH. Cada uno de los profesionales emplea una modalidad de evaluación propia, en donde no se verifica una estandarización de los procesos.

Ninguno de los participantes indicó seguir un protocolo o procedimiento específico para realizar la evaluación y diagnóstico en niños que presentan signos y síntomas asociados a este trastorno. Por otra parte, ningún participante indicó realizar un seguimiento del diagnóstico de TDAH ni observó una remisión de la sintomatología, aspecto que se atribuye a las características mismas del trastorno tal como éste se presenta en el DSM-5.

También nos parece importante mencionar que dentro del grupo de 21 psicólogos que trabajan con niños que han sido diagnosticados con este trastorno, solamente tres hacen referencia al uso de baterías psicológicas estandarizadas para medir los rasgos del trastorno TDAH, en consideración a Thurstone y Yela (2012): como las Escalas de Evaluación del Déficit de Atención con Hiperactividad (EDAH), o el Test de Percepción de Diferencias (CARAS), que se emplea para evaluar las aptitudes de atención y percepción.

Mencionando a Meneres-Sancho, Delgado-Pardo, Aires-González y Moreno-García (2015b): ninguno de los participantes menciona la aplicación de Test de Ejecución Continua, que se presenta actualmente como instrumento objetivo, actualizado y útil en su procedimiento, y que permite discriminar entre sujetos que presentan o no TDAH.

En cuanto al enfoque teórico-clínico de los participantes, se observó que los profesionales del enfoque psicoanalítico evalúan a sus pacientes a través 
de la aplicación de test proyectivos, como el Test Casa-Árbol-Persona (HTP) o el Test de Apercepción Infantil (CAT-A y CAT-H) y emiten un diagnóstico de TDAH a partir de la interpretación de resultados de dichas pruebas.

Por otra parte, es llamativo que algunos psicólogos, en particular aquellos que trabajan desde un enfoque humanista, afirman no proponer a sus pacientes un proceso formal de evaluación, aun cuando pronuncian el diagnóstico de TDAH con base en los criterios propuestos por el DSM-5.

En cuanto al tipo de herramientas de evaluación y diagnóstico que utilizan los psicólogos para diagnosticar el TDAH, todos los participantes enfatizan la importancia de la observación clínica (calidad del contacto con el niño, establecimiento del vínculo terapéutico y juego diagnóstico), seguido de la entrevista con los padres.

La observación del comportamiento del niño en el contexto de la entrevista clínica y la entrevista con los padres, aparecen como las fuentes privilegiadas por los profesionales ecuatorianos al momento de emitir el diagnóstico de TDAH. Sin embargo, este estudio nos permite constatar la importancia de la aplicación de baterías psicológicas objetivas y estandarizadas que sustenten el pronunciamiento de este diagnóstico.

Además, muy pocos psicólogos dan paso a la entrevista con los docentes o visitan las instituciones educativas, y admiten que no siempre ejecutan los protocolos emitidos por el Ministerio de Educación por considerarlos de poca utilidad para el proceso diagnóstico. Afirman también que reciben regularmente informes de los docentes y psicólogos escolares en los que se consignan quejas acerca de los niños, pero no se evidencian ni el seguimiento psicopedagógico realizado ni las adaptaciones escolares establecidas.

De manera indirecta, el presente estudio nos alerta acerca de la situación de los niños con este diagnóstico en las instituciones educativas. Varios participantes afirman que en muchos casos este diagnóstico promueve 
la segregación de los niños en la escuela. Podemos considerar que esta constatación compromete el proceso diagnóstico como tal, al sesgar la posición del psicólogo en función de los eventuales perjuicios éste que representaría para el paciente.

Los participantes constatan además que los padres de familia tienden a negar el diagnóstico en un primer momento, y en muchos casos el pronunciamiento del diagnóstico no representa ningún aporte para el proceso psicoterapéutico. Muy por el contrario, contribuye a la frustración de los padres y a la pérdida de referencias en cuanto a su rol en el desarrollo de sus hijos.

En las respuestas consignadas también se puede observar que varios profesionales, seis conocen y dan la importancia debida a la categorización de los síntomas y distinguen además los diferentes tipos de hiperactividad clasificados por el DSM-5. Para ellos, la correcta realización del proceso de evaluación y diagnóstico determina la posibilidad de elaborar un plan psicoterapéutico adecuado, tanto para el niño como para los padres.

Otro dato que nuestra investigación arroja es que el posicionamiento del psicólogo en cuanto al diagnóstico de TDAH depende, en gran medida, del enfoque teórico que fundamenta su trabajo clínico. Los psicólogos de orientación humanista, integrativo y psicoanalítico sostienen que la evaluación y diagnóstico no son indispensables para la elaboración de un plan psicoterapéutico.

Asimismo, la especialización del psicólogo ofrece una visión más o menos amplia en cuanto a la percepción del problema. Los psicólogos clínicos y los psicólogos infanto-juveniles tienden a otorgar más importancia a las manifestaciones clínicas del TDAH y a elaborar planes más innovadores en el plano terapéutico. Los psicólogos educativos y generales no se aventuran en el terreno del diagnóstico, que muchos de ellos consideran una tarea de los psicólogos especializados en estas temáticas.

Asimismo, el estudio demuestra una tendencia activa por parte de los 
psicólogos a realizar interconsultas con neurólogos-pediatras, con miras a confirmar o descartar el diagnóstico de TDAH a través de pruebas de imagen cerebral, como electroencefalogramas o resonancias magnéticas. Sin embargo, el estudio revela también que pocos neurólogos reenvían a los niños a la psicoterapia y realizan escasos aportes a la construcción conjunta de planes de tratamiento. Prevalece una alta medicalización como única medida terapéutica. Aun cuando existen progresos en el ámbito de la psicopatología infantil, este estudio demuestra que la intervención del psicólogo continúa siendo subestimada en el Ecuador.

El análisis de los resultados vuelve indiscutible la afirmación de que en nuestro país no existe un conocimiento suficientemente profundo acerca del proceso de evaluación y diagnóstico del TDAH, aun cuando se trata de una entidad clínica con alta incidencia y cuyo pronóstico muchas veces compromete los logros académicos y sociales a lo largo de toda la vida del paciente.

El presente estudio permite verificar que hay un acceso limitado a métodos de evaluación a partir de pruebas objetivas entre los profesionales que trabajan con una población infantil, en particular en las instituciones educativas y centros de salud públicos, en donde la demanda de atención psicológica supera largamente la capacidad de respuesta. Añadiremos que apenas un reducido porcentaje de familias puede tener acceso a un proceso psicoterapéutico privado para sus hijos en nuestro país.

De este modo, el TDAH continúa siendo abordado como una forma de mal comportamiento del niño, lo que deja un amplio margen a la aplicación de las referencias subjetivas de cada terapeuta, pasando enteramente por alto la complejidad de la sintomatología que el niño pone de manifiesto a través de conductas que, visiblemente, escapan al control del entorno. 


\section{Conclusiones}

En el Ecuador, los profesionales no cuentan con herramientas adecuadas o suficientes para realizar un diagnóstico de TDAH. El escaso apoyo de las instituciones públicas y la falta de directrices a nivel del sistema de educación nacional que favorezcan la inclusión de niños con dificultades de atención y aprendizaje es parte del problema.

El psicólogo podría beneficiarse al establecer nuevas formas de trabajo diagnóstico en las que se tengan en cuenta estas limitaciones. Privilegiar el trabajo en equipo, la comunicación con docentes y padres de familia y la discusión de resultados a nivel de una red de intervención coordinada, podría representar una solución viable y de largo alcance para favorecer un adecuado abordaje del TDAH. Nuevos estudios acerca del tema podrían apuntar a la construcción conjunta de soluciones, en las cuales la protección del niño y el apoyo a la familia constituyan los principales ejes de investigación.

Consideramos que el trabajo psicoterapéutico con niños debería implicar en todos los casos un enfoque interdisciplinario, en el que se tenga en cuenta el rol de los educadores, docentes y psicólogos escolares, se consideren y descarten problemáticas orgánicas o neurológicas y se planteen soluciones a partir de esta colaboración, que vendría a ofrecer además un apoyo concreto a los padres.

Las modalidades de evaluación y diagnóstico construidas a partir de esta recomendación podrían constituir incluso una medida de protección del niño en el ámbito escolar y familiar en nuestro país. El apoyo otorgado a los padres y a los docentes desde la posición del psicólogo, podría pensarse como una forma de garantizar el respeto de los derechos del niño. Al mismo tiempo, reconocemos la necesidad de que entidades gubernamentales e instituciones públicas y privadas unan esfuerzos para la concientización de esta problemática social, puesto que trasciende el marco de la consulta psicológica.

Podemos concluir que la labor del psicólogo no debería continuar 
siendo solitaria y aislada. El plan de tratamiento aplicado de forma independiente puede generar buenos resultados; sin embargo, es insuficiente para plantear una respuesta global satisfactoria en el ámbito emocional, escolar y familiar.

Añadiremos para terminar que las evaluaciones objetivas son, efectivamente, primordiales para emitir un adecuado diagnóstico, pero no ignoramos que el proceso psicoterapéutico también se fundamenta en el vínculo entre el paciente y el profesional. Algunos de los psicólogos participantes en el estudio mencionan haberse visto en la necesidad de desarrollar sus propias técnicas y estilos de tratamiento, mismos que son compatibles con los presupuestos teóricos propios de cada enfoque clínico.

Observamos entonces que existen intervenciones multimodales y que a nivel del tratamiento la cuestión se vuelve más amplia. Algunos pacientes obtienen resultados satisfactorios, inclusive de manera indirecta. Si bien la remisión de síntomas de TDAH no se verifica, varios profesionales refieren una mejoría en la esfera relacional, familiar y emocional a través del tratamiento psicoterapéutico.

\section{Referencias}

Asociación Americana de Psiquiatría (2014a,b). Manual diagnóstico y estadístico de los trastornos mentales (DSM-5®). $5^{\text {a }}$ Edición, ISBN: 978-84-9835-810-0. España: Editorial Medica Panamericana.

Benedet, M. (2002). Neuropsicología cognitiva: Aplicaciones a la clínica y a la investigación del fundamento teórico y metodológico de la neuropsicología cognitiva. 1ra edición, ISBN: 84-8446-047-9. Madrid, España: Instituto de Migraciones y Servicios Sociales (IMSERSO).

Claro, S. (2015). Luche-Mundo: Una metodología para el abordaje responsable y dialógico del TDAH. Estudios Pedagógicos, 41(Número especial), 31-50, e-ISSN: 0718-0705. Recuperado de: 
https://doi.org/10.4067/S0718-07052015000300003

CONA (2013). Código de la Niñez y Adolescencia. Ley No. 2002-100. Ecuador: Congreso Nacional.

Hidalgo, M., \& Sánchez, L. (2014). Trastorno por déficit de atención e hiperactividad. Manifestaciones clínicas y evolución. Diagnóstico desde la evidencia científica. Pediatría Integral, 18(9), 609-623, eISSN: 1135-4542. España: Ediciones Ergon, S.A.

Manrique, A. (2019). Acciones Pedagógicas para la Atención de Niños/as con Trastorno por Déficit de Atención e Hiperactividad (TDAH). Revista Scientific, 4(11), 46-66, e-ISSN: 2542-2987. Recuperado de: https://doi.org/10.29394/Scientific.issn.2542-2987.2019.4.11.2.46-66 Meneres-Sancho, S., Delgado-Pardo, G., Aires-González, M. \& MorenoGarcía, I. (2015a,b). Tests de ejecución continua: Integrated Visual and Auditory Continuous Performance Test (IVA/CPT) y TDAH. Una revisión. Revista de Psicología Clínica con Niños y Adolescentes, 2(2), 107-113, e-ISSN: 2340-8340. Recuperado de:

https://www.redalyc.org/articulo.oa?id=477147186003

Milla, M. (2018). Repercusiones del TDAH en el alumnado de Educación Infantil. Trabajo fin de Grado. España: Universidad de Jaén. Recuperado de: https://hdl.handle.net/10953.1/8177 MINEDUC (2016). Instructivo para la evaluación y promoción de estudiantes con necesidades educativas especiales. JA/AS/BM/CV. Ecuador: Ministerio de Educación.

OSE (2019a,b). Situación de la niñez y adolescencia en Ecuador: Una mirada a través de los ODS. Ecuador: Observatorio Social del Ecuador.

Ramos, C., Bolaños, M., \& Ramos, D. (2015). Prevalencia del Trastorno por Déficit de Atención con Hiperactividad en Estudiantes Ecuatorianos. Revista Científica y Tecnológica UPSE, 3(1), 13-19, e- 
ISSN: 1390-7697. Recuperado de: https://doi.org/10.26423/rctu.v3i1.72 Reza, L., Solórzano, M., Erazo, E., Oñate, F., \& Lamingo, G. (2018). EI TDAH y su repercusión en el rendimiento académico. Revista Atlante: Cuadernos de Educación y Desarrollo, 1-19, e-ISSN: 1989-4155. España: Universidad de Málaga (UMA).

Thurstone, L., \& Yela, M. (2012). CARAS-R: Test de percepción de diferencias - Revisado. 11. a edición, revisada y ampliada, ISBN: 97884-15262-65-7. Madrid, España: TEA Ediciones, S.A.U.

Zuluaga, J., \& López, E. (2017). Evolución del estilo cognitivo en niños con TDAH bajo tres tipos de intervención junto a un grupo sin ningún tipo de tratamiento. Katharsis, (24), 33-52, e-ISSN: 2500-5731. Recuperado de:

http://revistas.iue.edu.co/index.php/katharsis/article/view/964 
Artículo Original / Original Article

\section{Estefanía Isabel Lara Orozco}

e-mail: eilara.mpp@uisek.edu.ec

Nacida en Riobamba, Ecuador, el 29 de septiembre

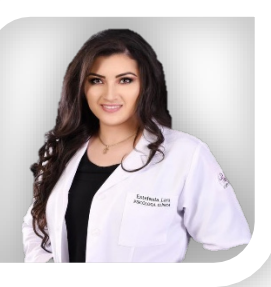
del año 1989. Psicóloga clínica por la Pontificia Universidad Católica del Ecuador (PUCE); Magíster en Psicología con mención en Psicoterapia por la Universidad Internacional SEK (UISEK); Psicóloga en el Centro Médico Metropolitano de la ciudad de Riobamba y especialista en atención psicológica a niños y adolescentes. 


\section{Gabriela Alexandra Pazmiño Márquez}

e-mail: gpazminom@gmail.com

Nacida en Quito, Ecuador, el 24 de junio del año 1984.

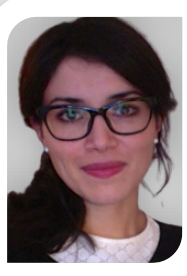

Psicóloga clínica por la Pontificia Universidad Católica del Ecuador (PUCE); Máster en Humanidades y Ciencias Sociales mención Psicoanálisis por la Université de Vincennes à Saint-Denis (UP8), Francia; Doctora en Psicopatología y Psicoanálisis por la Université Paris Diderot VII, Francia; Docente Titular de la carrera de Psicología de la Universidad Internacional SEK (UISEK), Quito, Ecuador; Experiencia clínica con niños y adolescentes; autora de varios artículos sobre psicopatología infantil. 


\section{Janethcia del Rocío Játiva Morillo}

e-mail: rocio.jativa@uisek.edu.ec

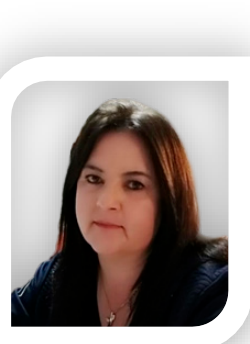

Nacida en Tulcán, Ecuador, el 15 de marzo del año 1968.

Licenciada en Psicología de la Universidad Central del

Ecuador (UCE); Doctora en Administración Educativa de

la Escuela Politécnica Javeriana (ESPOJ); Magister en Intervención y Terapia Familiar Sistémica por la Universidad Politécnica Salesiana (UPS); Experiencia en el ámbito educativo, docente y psicóloga de Institutos Militares; práctica clínica a favor de la niñez, adolescencia y de grupos de atención prioritaria en el Ministerio de Inclusión Económica y Social (MIES); Ministerio de Justicia Derechos Humanos y Cultos (MJDHC); Experiencia en Formación Inicial de Jueces, Fiscales y Defensores Públicos; Subdirectora de la Escuela de la Función Judicial, consulta privada; docente de maestría en la Universidad Internacional SEK (UISEK), Quito, Ecuador.

El contenido de este manuscrito se difunde bajo una Licencia de Creative Commons ReconocimientoNoComercial-Compartirlgual 4.0 Internacional 